\title{
Téoros
}

Revue de recherche en tourisme

\section{Le potentiel des voyageurs américains en matière de tourisme culinaire}

\section{Maïthé Levasseur}

Volume 26, numéro 2, été 2007

URI : https://id.erudit.org/iderudit/1070949ar

DOI : https://doi.org/10.7202/1070949ar

Aller au sommaire du numéro

Éditeur(s)

Université du Québec à Montréal

ISSN

0712-8657 (imprimé)

1923-2705 (numérique)

Découvrir la revue

Citer cette note

Levasseur, M. (2007). Le potentiel des voyageurs américains en matière de tourisme culinaire. Téoros, 26(2), 71-73. https://doi.org/10.7202/1070949ar d'utilisation que vous pouvez consulter en ligne.

https://apropos.erudit.org/fr/usagers/politique-dutilisation/ 


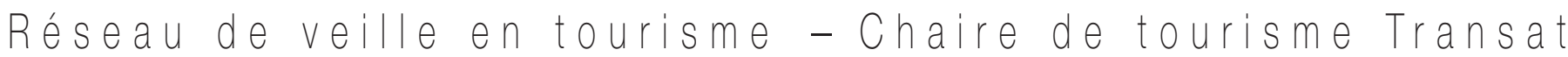 \\ Le potentiel des voyageurs américains en matière de tourisme culinaire}

\section{Maïthé Levasseur}

Le tourisme culinaire se développe rapidement depuis quelques années et intéresse les intervenants touristiques de nombreuses destinations. Une récente étude américaine confirme la présence d'une niche importante de touristes culinaires aux États-Unis. On estime que $17 \%$ des touristes d'agrément ont participé à une ou plusieurs activités liées à la gastronomie et au vin au cours des trois dernières années, constituant la clientèle de touristes culinaires. L'étude permet de découvrir les différents types de touristes culinaires, leur profil ainsi que leurs intérêts.

Le tourisme culinaire va au-delà de l'expérience de la fine cuisine. Selon la CCT (Commission canadienne du tourisme, 2002), «il comprend les activités culinaires, agrotouristiques et agroalimentaires spécialement conçues pour les touristes, qui mettent en valeur les aliments et les boissons et qui fournissent l'occasion de découvrir les plats et vins propres à une région et de reconnaître le talent et l'ingéniosité des artisans ». Le tourisme culinaire devient très tendance. "La recherche d'activités nouvelles, l'envie de découvrir d'autres cultures, de donner du sens et d'enrichir ses vacances sont au cœur des attentes touristiques d'aujourd'hui, notamment dans le tourisme haut de gamme ", selon Caroline Puechoultres, directrice du marketing stratégique du Club Med (Stratégies.fr, 2005).

\section{Les différents types de touristes culinaires}

L'étude de la Travel Industry Association (TIA, 2007) identifie trois segments parmi les touristes américains qui apprécient la gastronomie ou le vin durant un voyage:

- délibérés: touristes qui ont fait au moins un voyage où les activités liées à la gastronomie et au vin étaient la principale raison du séjour ou avaient influencé le choix de la destination ;

- opportunistes : touristes qui ont fait au moins un voyage qui incluait des activités associées à la gastronomie et au vin, mais où ces activités n'étaient pas un facteur de décision de la destination;

- accidentels : touristes qui ont fait au moins un voyage où ils ont participé à des activités liées à la gastronomie et au vin, simplement parce que ces activités étaient disponibles.

La TIA estime à 27,3 millions le nombre de touristes culinaires aux États-Unis (tableau 1). Les segments les plus intéressants à considérer sont toutefois les touristes culinaires délibérés et opportunistes, qui représentent 12,5\% du total des touristes d'agrément.

\section{Tableau 1}

Estimation du nombre de touristes culinaires aux États-Unis en 2006

\begin{tabular}{lcc} 
Type de touristes culinaires & $\begin{array}{c}\text { Part des touristes } \\
\text { d'agrément }\end{array}$ & $\begin{array}{c}\text { Part de la population } \\
\text { (millions) }\end{array}$ \\
\hline Délibérés & $7,8 \%$ & 12,6 \\
Opportunistes & $4,7 \%$ & 7,6 \\
Accidentels & $4,4 \%$ & 7,1 \\
Total & $16,9 \%$ & 27,3
\end{tabular}

Source : TIA, 2007.

De ce nombre, $10 \%$ sont davantage intéressés par les activités qui se rapportent à la gastronomie et $9 \%$ par celles reliées au vin (schéma 1).

\section{Schéma 1}

Distribution et taille du marché des touristes culinaires américains

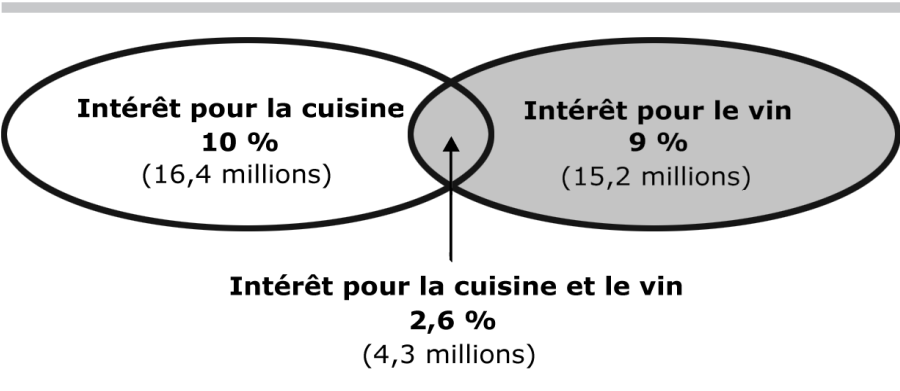

Source : TIA, Profile of Culinary Travelers, 2006 Edition.

Dans la présente analyse, nous ne retiendrons que l'information concernant les touristes culinaires délibérés et opportunistes.

\section{Le profil des touristes culinaires}

Le profil du touriste culinaire ne diffère pas de façon significative de celui des autres touristes d'agrément pour ce qui concerne le sexe ou l'âge de la clientèle. II est néanmoins intéressant de constater que $53 \%$ ont moins de 44 ans (graphique 1).

Il y a par contre des différences plus marquées parmi les groupes socioéconomiques. En effet, les touristes culinaires ont un niveau de scolarité ainsi qu'un revenu plus élevés que l'ensemble des touristes d'agrément américains (graphique 2). 


\section{Graphique 1}

Répartition des touristes culinaires et non culinaires selon le sexe et l'âge

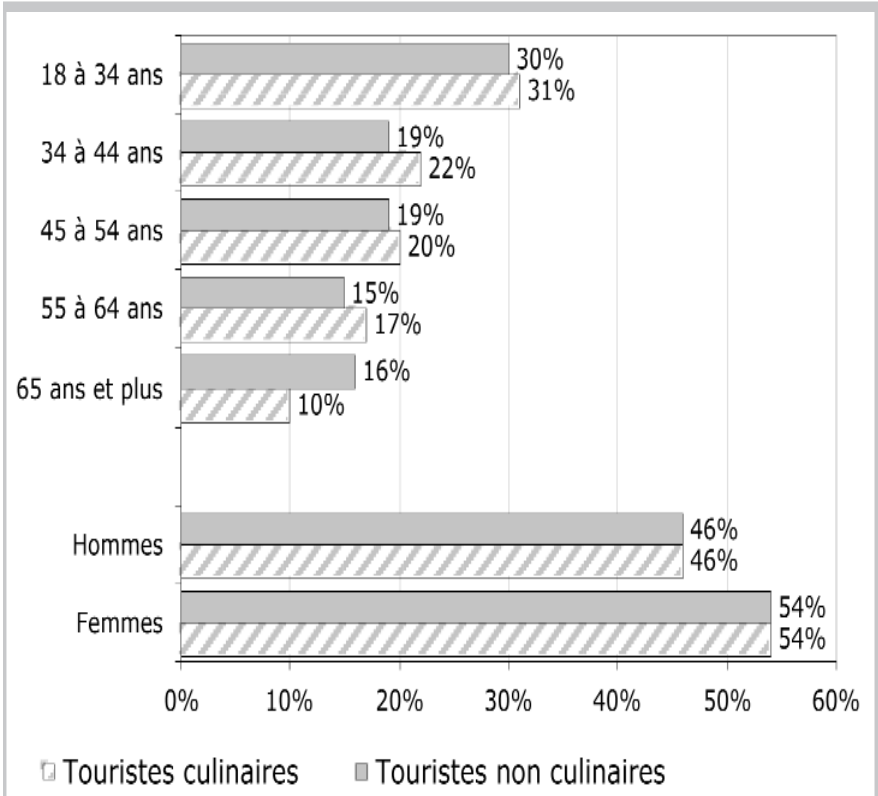

Source: TIA.

\section{Graphique 2}

Répartition des touristes culinaires et non culinaires selon la scolarité et le revenu

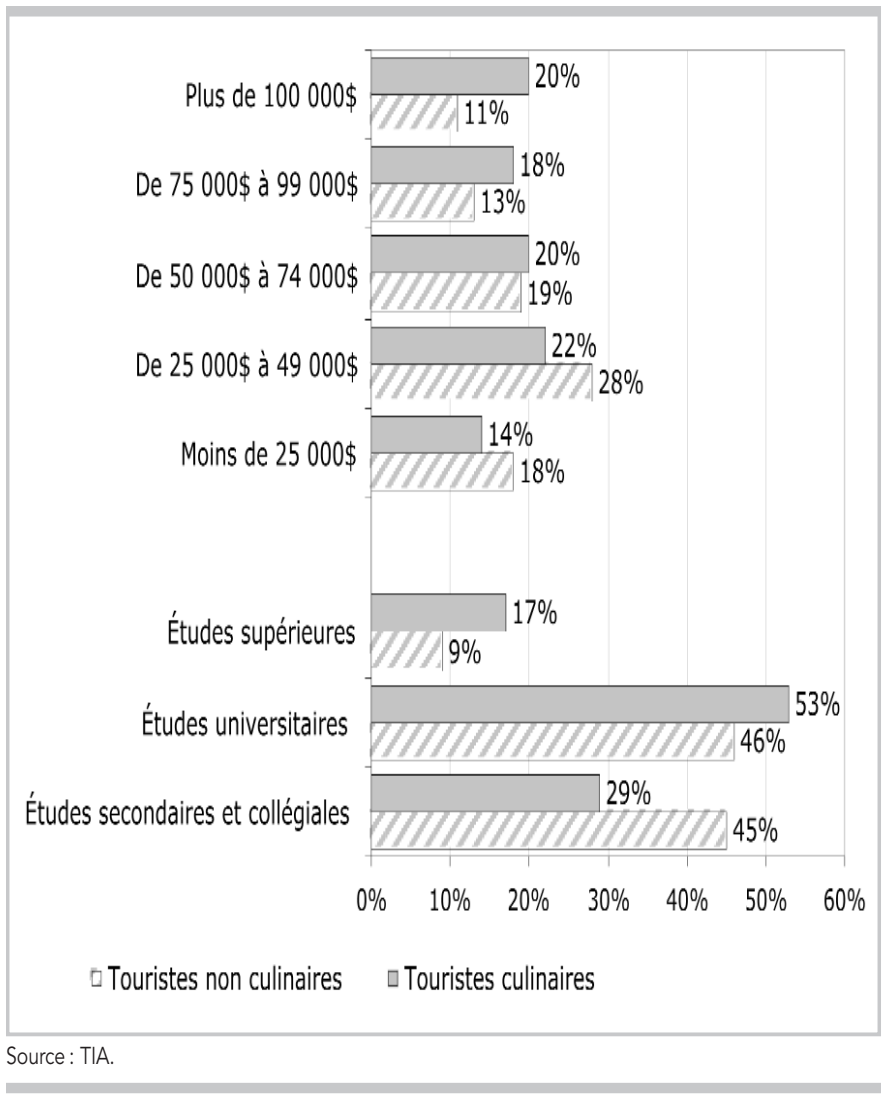

Les touristes culinaires sont très actifs et participent à de nombreuses autres activités au cours d'un séjour dans une proportion plus forte que les touristes non culinaires. Le graphique 3 illustre que la participation des touristes culinaires à diverses activités est plus forte. Ils sont particulièrement intéressés par les activités culturelles, ils apprécient les spas et visitent les parcs nationaux et les sites historiques.

\section{Graphique 3}

Activités pratiquées par les touristes culinaires et non-culinaires

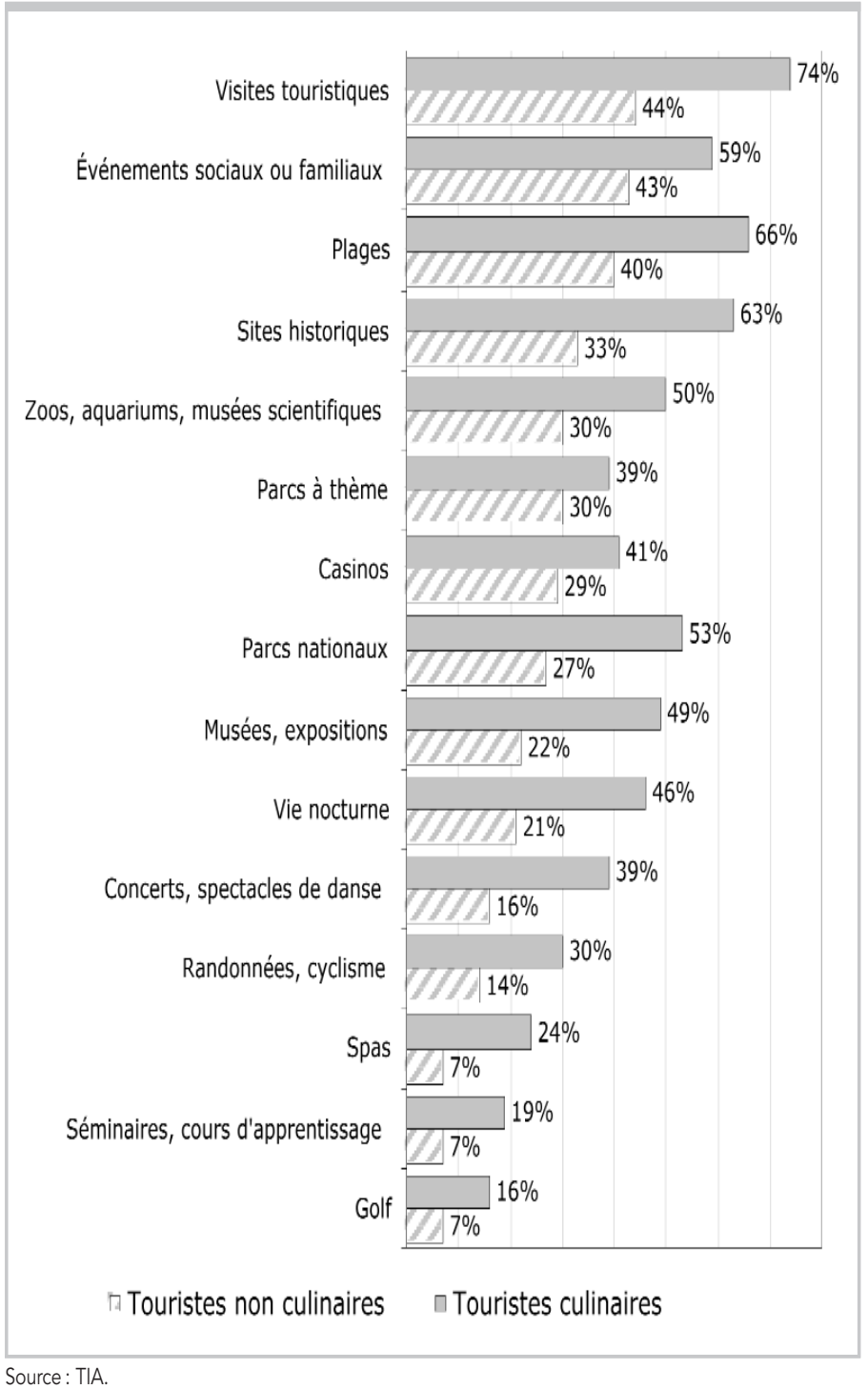

Grands lecteurs, ils sont plus sensibles que les autres touristes d'agrément à toutes les publications testées par l'étude, notamment les cahiers spéciaux des journaux, les publications culinaires, les magazines de voyages et, bien sûr, les médias qui traitent de gastronomie et de vin. Ils consultent davantage Internet et utilisent les alertes Web. Les magazines spécialisés sont donc un média pertinent pour rejoindre cette clientèle, tout comme l'information en ligne. 
Les principaux facteurs influençant le choix d'une destination sont l'expérience gastronomique unique et différente, la possibilité d'expérimenter une cuisine différente de chez soi, l'atmosphère originale et l'accessibilité des restaurants. Puisque vivre une expérience unique est le facteur se démarquant le plus fortement, il est important de l'inscrire dans une destination qui a du caractère et qui présente d'autres éléments attractifs (environnement physique, attraits culturels...).

Les touristes culinaires risquent d'être sensibles à des forfaits qui mettront l'accent sur l'unicité de l'expérience dans sa globalité, pas seulement sous l'angle gastronomique. Les destinations et les organisateurs de voyages devraient donc travailler conjointement afin d'offrir une «immersion» dans la culture et la gastronomie locales. Cette immersion devrait toucher autant l'hébergement que l'offre complémentaire et les activités liées à la gastronomie et au vin. Les forfaits incitent les visiteurs à prolonger la durée de leur séjour et à visiter un plus grand nombre d'attraits.

En comparaison avec les autres touristes d'agrément, les touristes culinaires ont beaucoup plus tendance à rapporter des produits ou des vins locaux avec eux pour les partager avec leur famille et leurs amis. II s'agit d'une autre occasion que les destinations devraient saisir.

\section{Les particularités des amateurs de bonne cuisine et des amateurs de vin}

La plupart des touristes qui réalisent des activités liées à la gastronomie voyagent accompagnés (87\%), mais une part surprenante (25\%) de ceux-ci voyagent en compagnie d'amis ou de collègues plutôt que de membres de leur famille. Les amateurs de bonne cuisine accordent environ un tiers de leur budget de voyage à des activités qui touchent la gastronomie et cette proportion est plus forte pour les touristes délibérés (34\%) et opportunistes (50\%).

De leur côté, les amateurs de vin visitent souvent (51\%) plus d'un vignoble au cours de leur voyage (70\% des touristes délibérés). Les activités liées au vin représentent environ $23 \%$ de leur budget (comparativement à $36 \%$ dans le cas des touristes culinaires délibérés). Pour ces touristes américains, il n'est pas étonnant d'apprendre que la Californie est de loin la destination préférée (32 \%).

\section{Le potentiel de croissance}

\section{Tableau 2}

Estimation du nombre d'amateurs de bonne cuisine et d'amateurs de vin aux États-Unis en 2006

\begin{tabular}{lcc} 
Type de touristes culinaires & Intérêt pour la cuisine & Intérêt pour le vin \\
\hline Délibérés & $4,8 \%(7,9$ millions $)$ & $4,1 \%(6,6$ millions $)$ \\
Opportunistes & $3,0 \%(4,9$ millions $)$ & $2,6 \%(4,2$ millions $)$ \\
Accidentels & $2,3 \%(3,7$ millions $)$ & $2,7 \%(4,4$ millions $)$ \\
Total & $10,1 \%(16,5$ millions $)$ & $9,4 \%(15,2$ millions $)$ \\
\hline Source : TIA, 2007. & & \\
\hline
\end{tabular}

Le tourisme culinaire présente un excellent potentiel de croissance pour les prochaines années. Non seulement l'offre se développe et se raffine, mais le nombre d'adeptes grandit également. Selon l'étude ci-dessus, $60 \%$ des touristes d'agrément américains se disent intéressés à participer à des activités qui gravitent autour de la gastronomie ou du vin au cours d'un prochain voyage. Les activités gastronomiques ou liées au vin qui les intéressent sont: vivre une expérience unique dans un restaurant, découvrir la cuisine locale, visiter des fermes agrotouristiques, participer à des festivals culinaires, déguster des vins locaux ou faire une tournée de vignobles.

\section{L'offre touristique culinaire au Québec}

Avec l'apparition de la nouvelle cuisine conjuguée à la créativité de ses chefs, le Québec affirme une personnalité touristique bien distincte grâce à ses produits du terroir. L'organisation de l'offre touristique québécoise pour proposer des expériences culinaires est amorcée depuis plusieurs années. La Route des saveurs dans Charlevoix, la Route des vins dans les Cantons-de-l'Est et la Route des fromages en sont de bons exemples.

Les Québécois sont d'ailleurs des amateurs de fine cuisine ; $30 \%$ de la population fréquente occasionnellement les restaurants haut de gamme, pas nécessairement lors d'un séjour touristique (Print Measurement Bureau, 2006). Toutefois, ces derniers ne se démarquent pas du reste de la population canadienne où la moyenne est de $34 \%$. Plus spécifiquement à Montréal, $36 \%$ de la population fréquente les restaurants haut de gamme, favorisant ainsi la popularité d'événements tels que le Festival Montréal en Lumière. En effet, le volet culinaire du festival se positionne comme le plus important événement gastronomique d'Amérique du Nord. Les soirées gastronomiques où de grands chefs sont invités, les activités au Marché Jean-Talon et la Fête des fromages d'ici en 2007 ont permis de consolider la notoriété de Montréal en tant que destination gourmande.

Le tourisme culinaire au Québec fait donc ses marques par l'intermédiaire d'une offre variée ainsi que de divers événements et circuits. La clientèle actuelle est majoritairement québécoise, mais le potentiel pour attirer la clientèle américaine est bien présent.

Maïthé Levasseur est analyste au Réseau de veille en tourisme de l'Université du Québec à Montréal.

\section{Bibliographie}

Commission canadienne du tourisme (2002), Cultiver le goût du tourisme culinaire : une stratégie de développement de produits, juin.

Lejeune-Piat, Marie (2007), "Fourneaux: le tourisme culinaire fait recette", Stratégies, [http://www.strategies.fr/archives/1363_2/1363_201201], consulté le 31 mars 2007.

Print Measurement Bureau (2006), Two-Year Readership Database.

Travel Industry Association (2007), Profile of Culinary Travelers, édition 2006. 